\title{
On Mechanistic Explanation of the Shape of the Universal Curve of Earthquake Recurrence Time Distributions
}

\author{
Mariusz BIAŁECKI \\ Institute of Geophysics, Polish Academy of Sciences, Warszawa, Poland \\ e-mail: bialecki@igf.edu.pl
}

\begin{abstract}
This paper outlines an idea for an explanation of a mechanism underlying the shape of the universal curve of the Earthquake Recurrence Time Distributions. The proposed simple stochastic cellular automaton model is reproducing the gamma distribution fit with the proper value of parameter $\gamma$ characterizing the Earth's seismicity and also imitates a deviation from the fit at short interevent times, as observed in real data.

Thus the model suggests an explanation of the universal pattern of rescaled Earthquake Recurrence Time Distributions in terms of combinatorial rules for accumulation and abrupt release of seismic energy.
\end{abstract}

Key words: stochastic cellular automaton, earthquake recurrence time, avalanches, toy model of earthquakes, Markov chains.

\section{INTRODUCTION}

Analyzing seismic catalogs, Corral (2004) has determined that the probability densities of the waiting times between earthquakes for different spatial areas and magnitude ranges can be described by a unique universal distribution if the time is rescaled with the mean rate of occurrence. 
To unify diverse observations, the spatiotemporal analysis was carried out as follows. Seismicity is considered as a result of a dynamical process, whose collective properties are largely independent of the physics of individual earthquakes. Following Bak et al. (2002), events are neither separated into different kinds (foreshocks, mainshocks, aftershocks) nor the crust is divided into provinces with different tectonic properties. Then, a region of the Earth is selected, as well as temporal period and a minimum magnitude $M_{c}$ (for conditions and other details, see Corral $(2004,2007))$. Events in this space-timemagnitude window are considered as a point process in time (disregarding the magnitude and the spatial degrees of freedom) and are characterized only by their occurrence time $t_{i}$, with $i=1 \ldots N\left(M_{c}\right)$. Then the recurrence (or waiting) time $\tau_{i}$ is defined by $\tau_{i}=t_{i}-t_{i-1}$.

The entire Earth has been analyzed by this method and it appears that different regions' probability densities of waiting times, rescaled by the mean seismic rate, as a function of the rescaled recurrence time, collapse onto a single curve $f$ (Corral 2004):

$$
D\left(\tau ; M_{c}\right)=R\left(M_{c}\right) f\left(R\left(M_{c}\right) \tau\right),
$$

where mean seismic rate $R\left(M_{c}\right)$ is given by $R\left(M_{c}\right)=N\left(M_{c}\right) / T$ (here $T$ is a total time into consideration), and recurrence-time probability density $D\left(\tau ; M_{c}\right)$ is defined as $D\left(\tau ; M_{c}\right)=\operatorname{Prob}[\tau<$ recurrence time $<\tau+d \tau] / \tau$. The so-called scaling function $f$ admits a fit in the form of a generalized gamma distribution

$$
f^{f i t}(\theta)=C \theta^{\gamma-1} \exp \left(-\frac{\theta^{\delta}}{\beta}\right),
$$

where $\gamma=0.67 \pm 0.005, \beta=1.58 \pm 0.15, \delta=0.98 \pm 0.05, C=0.5 \pm 0.1$, and $\theta=R \tau$ is dimensionless recurrence time. The value of $\delta$ can be approximated to 1 . The present characterization of the stochastic spatiotemporal occurrence of earthquakes by means of a unique law would indicate the existence of universal mechanisms in the earthquake-generation process (Corral 2004).

This paper outlines an idea for explanation of a possible mechanism underlying the shape of the universal curve in terms of a mechanistic model, namely a cellular automaton model called Random Domino Automaton (RDA). The simple rules for evolution of the model, being a slowly driven system, rely on accumulation and abrupt release of energy only, which well fits the abovedescribed procedure of neglecting individual properties of earthquakes as well as diversified tectonic conditions. We show that RDA reproduces the shape of the "rescaled" distribution of recurrence times.

As can be seen from the original work (Corral 2004) as well as from further studies (Marekova 2012), results obtained from various earthquake catalogs show a deviation from the gamma distribution at the short interevent times. 
This holds from worldwide to local scales and for quite different tectonic environments. It is remarkable that the presented toy model reproduces also this deviation. Thus the model suggests an explanation of the universal pattern of rescaled Earthquake Recurrence Time Distributions in terms of its combinatorial rules for accumulation and release of seismic energy.

Note, that we present a preliminary results - here we are not covering the realistic range of earthquake energies. So far, some insight into the origin of the gamma distribution as well as examination the recurrence statistics of a range of cellular automaton earthquake models are presented in Weatherley (2006). It is shown there that only one model, the Olami-Feder-Christensen automaton, has recurrence statistics consistent with regional seismicity for a certain range of conservation parameters of that model.

An explanation of the earthquake recurrence times in terms of probability distributions is presented by Saichev and Sornette (2006, 2013).

\section{RANDOM DOMINO AUTOMATON}

The Random Domino Automaton (RDA) was introduced as a toy model of earthquakes (Białecki and Czechowski 2010, 2013, 2014; Białecki 2015), but can be also regarded as an extension of the well-known 1-D forest-fire model proposed by Drossel and Schwabl (1992). As a field of application of RDA we have already studied its relation to Ito equation (Czechowski and Białecki 2012a,b) and to integer sequences (Białecki 2012). We point out also other cellular automata models (Tejedor et al. 2009, 2010) giving an insight into diverse specific aspects of seismicity.

The RDA is characterized as follows:

- space is 1-dimensional and consists of $N$ cells; periodic boundary conditions are assumed;

- cell may be empty or occupied by a single ball;

- time is discrete and in each time step an incoming ball hits one arbitrarily chosen cell (the same probability for each one). The balls are interpreted as energy portions.

The state of the automaton changes according to the following rule:

- if the chosen cell is empty, it becomes occupied with probability $\nu$; with probability $(1-\nu)$ the incoming ball is rebounded and the state remains unchanged;

- if the chosen cell is occupied, the incoming ball provokes an avalanche with probability $\mu$ (it removes balls from hit cell and from all adjacent cells); with probability $(1-\mu)$ the incoming ball is rebounded and the state remains unchanged.

The parameter $\nu$ is assumed to be constant but the parameter $\mu=\mu_{i}$ is allowed to be a function of size $i$ of the hit cluster. This extension with respect 
to Drossel-Schwabl model leads to substantial novel properties of the automaton. Note that only the ratio of these parameters, $\mu_{i} / \nu$, affects properties of the automaton - changing of $\mu$ and $\nu$ proportionally corresponds to a rescaling of time unit.

The remarkable feature of the RDA is the explicit one-to-one relation between details of the dynamical rules of the automaton (represented by rebound parameters $\left.\mu_{i} / \nu\right)$ and the produced stationary distribution $n_{i}$ of clusters of size $i$, which implies distribution of avalanches $w_{i}$. It shows how to reconstruct details of the "macroscopic" behavior of the system from simple rules of "microscopic" dynamics.

Various sizes $N$ of RDA can be considered in order to explain the shape of the universal curve of Scaling Law. It appears that the results for quite a small size $N=5$ are enough to explain the idea and allow to keep the reasoning concise and detailed. RDA for a bigger size of the lattice behaves similar, and the overall picture remains the same, as results from explanations given below.

RDA is also a Markov chain (Białecki 2015). It is convenient to define states $i$ up to translational equivalence. Thus, in the example for $N=5$, instead of $2^{5}$, there are 8 states only - see Table 1 . Such a space of states is irreducible, aperiodic and recurrent. Transition matrix $\mathbf{p}$, where $[\mathbf{p}]_{i j}=$ probability of transition $i \longrightarrow j$, for $N=5$ is of the form

$\frac{1}{5}\left(\begin{array}{cccccccc}5-5 \nu & 5 \nu & 0 & 0 & 0 & 0 & 0 & 0 \\ \mu_{1} & 5-\mu_{1}-4 \nu & 2 \nu & 2 \nu & 0 & 0 & 0 & 0 \\ 2 \mu_{2} & 0 & 5-2 \mu_{2}-3 \nu & 0 & 2 \nu & \nu & 0 & 0 \\ 0 & 2 \mu_{1} & 0 & 5-2 \mu_{1}-3 \nu & \nu & 2 \nu & 0 & 0 \\ 3 \mu_{3} & 0 & 0 & 0 & 5-3 \mu_{3}-2 \nu & 0 & 2 \nu & 0 \\ 0 & 2 \mu_{2} & \mu_{1} & 0 & 0 & 5-2 \mu_{2}-\mu_{1}-2 \nu & 2 \nu & 0 \\ 4 \mu_{4} & 0 & 0 & 0 & 0 & 0 & 5-4 \mu_{4}-\nu & \nu \\ 5 \mu_{5} & 0 & 0 & 0 & 0 & 0 & 0 & 5-5 \mu_{5}\end{array}\right)$

Table 1

States of RDA for the size of the lattice $N=5$

\begin{tabular}{|c|c|c|}
\hline State label & Example & Multiplicity \\
\hline 1 & $\hookrightarrow|||| \mid \leftarrow$ & 1 \\
\hline 2 & $\hookrightarrow|||||\bullet| \hookleftarrow$ & 5 \\
\hline 3 & $\hookrightarrow|||||\bullet| \bullet \mid \hookleftarrow$ & 5 \\
\hline 4 & $\hookrightarrow|||\cdot||\bullet| \hookleftarrow$ & 5 \\
\hline 5 & $\hookrightarrow|||\cdot| \bullet|\bullet| \hookleftarrow$ & 5 \\
\hline 6 & $\hookrightarrow \hookrightarrow|| \bullet|| \bullet|\bullet| \hookleftarrow$ & 5 \\
\hline 7 & $\hookrightarrow|| \cdot|\bullet| \cdot|\bullet| \hookleftarrow$ & 5 \\
\hline 8 & $\hookrightarrow|\bullet| \cdot|\bullet| \cdot|\bullet| \bullet \mid \hookleftarrow$ & 1 \\
\hline
\end{tabular}




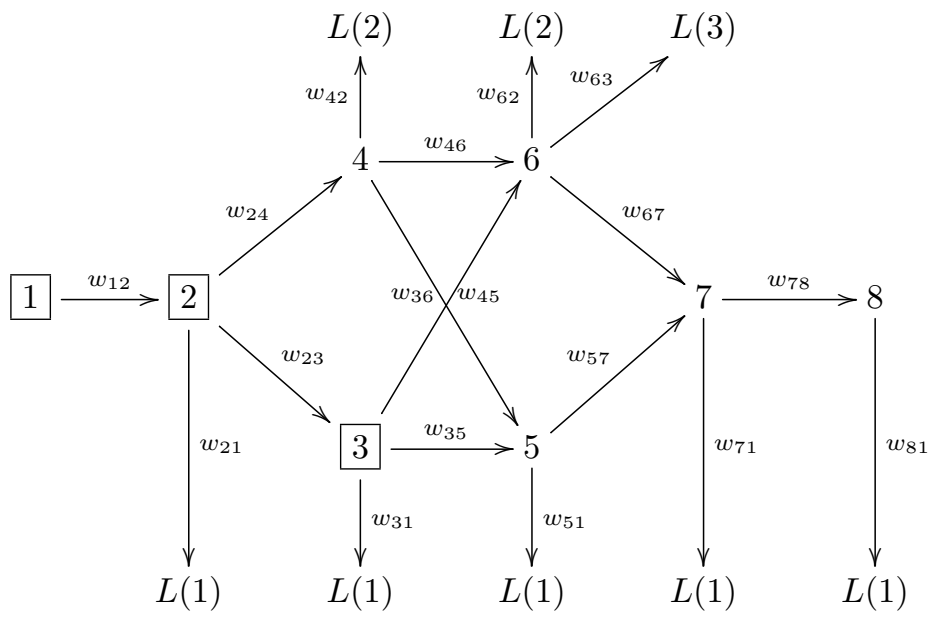

Fig. 1. A state diagram for RDA of size $N=5$. Arrows with respective weights indicate possible transitions; those with avalanches are ended with symbol "L". A state is boxed, if it is possible to get it directly after an avalanche.

Stationary distribution is given by

$$
v \cdot \mathbf{p}=v .
$$

The evolution of the system is represented in Fig. 1. Arrows between states $i$ and $j$, with respective weights $w_{i j}$, indicate possible transitions. A symbol $L(j)$ depicts an avalanche to state $j$. The density of the system is growing from left side (state 1 has density $\rho=0$ ) to right side (up to density $\rho=1$ for state 8$)$.

The expected time between two consecutive avalanches $T_{a v}$ may be expressed by various formulas (Białecki 2015). For example

$$
T_{a v}=\frac{\langle w\rangle+1}{1-P_{r}},
$$

where $\langle w\rangle$ is the average avalanche size and $P_{r}$ is the probability that the incoming ball is rebounded both from empty or occupied cell.

The probabilities $v_{i}$ of states $i$ obtained from condition 3 allow determining the distribution of frequency $f_{i}$ of avalanche of size $i$, if rebound parameters $\mu_{i} / \nu$ are given. There exists also a procedure of obtaining approximate values of rebound parameters $\mu_{i} / \nu$, which produce requested distribution of avalanches (Białecki 2013). The approximation comes from nonexistence of exact equations for (stationary) distribution of clusters $n_{i}$ for sizes bigger than 
4 (see Białecki 2015). We have used this procedure to obtain values of $\mu_{i} / \nu$ that give noncumulative inverse-power distribution of avalanches presented in Table 2, i.e., in the form consistent with Gutenberg-Richter law. The exact value of power (here 2.1) does not affect results of the construction substantially.

Table 2

Approximate values of rebound parameters $\mu_{i}$ and respective avalanche distribution $w_{i}$

\begin{tabular}{|l|c|c|c|c|c|}
\hline$i$ & 1 & 2 & 3 & 4 & 5 \\
\hline$\mu_{i}$ & 0.999060 & 0.388232 & 0.284504 & 0.097650 & 0.045810 \\
$w_{i}$ & 0.413247 & 0.102851 & 0.042587 & 0.022351 & 0.014306 \\
\hline
\end{tabular}

Note: The parameter $\nu=0.25$.

\section{DISTRIBUTION OF WAITING TIMES}

To calculate the distribution of waiting times, each path starting from a state reached after an avalanche and ending with an avalanche is considered. There are 42 such paths for size $N=5$. Each path is assigned its respective probabilities that a total passage time is equal to $1,2, \ldots$ time steps.

Respective weights $S_{k}$ describing how often the system starts from initial state $k$ are given by

$$
S_{k}=\frac{\sum_{i>k} v_{i} p_{i k}}{\sum_{k} \sum_{i>k} v_{i} p_{i k}} .
$$

For $N=5$ initial states are 1,2 and 3 .

The expected time of stay in a state $k$ is

$$
t_{a v}(k)=\sum_{i} p_{k k}^{(i-1)}\left(1-p_{k k}\right) \cdot i=\left(1-p_{k k}\right)^{-1} .
$$

The probability of stay in given state $k$ for a time equal to $i$ time steps is given by

$$
T_{i}^{k}=p_{k k}^{(i-1)}\left(1-p_{k k}\right),
$$

and all possible values are aggregated in a vector $T^{k}$ with $i$-th component equal to $T_{i}^{k}$. For a path through two consecutive states, $k$ and $l$, the respective probability of time of stay in both of them equal to $j$ time steps is defined by

$$
T_{j}^{k l}=\left(T^{k} \star T^{l}\right)_{j}=\sum_{n=1}^{j-1} T_{j}^{k} T_{n-j}^{l} .
$$


For a path through three states, $k, l$ and $m$ we have $T^{k l m}=\left(T^{k} \star T^{l}\right) \star T^{m}$, and so on for longer paths.

The probability rates $w_{i j}$ for transition $i \rightarrow j$, where $i \neq j$, are just normalized probabilities $p_{i j}$, namely

$$
w_{i j}=\frac{p_{i j}}{\sum_{j \neq i} p_{i j}} .
$$

Thus for a path $i_{1}, i_{2}, \ldots, i_{k-1}, i_{k}$ there is assigned a combined weight

$$
W^{i_{1} i_{2} \ldots i_{k-1} i_{k}}=S_{i_{1}} \cdot w_{i_{1} i_{2}} \cdot \ldots \cdot w_{i_{k-1} i_{k}},
$$

as well as combined weigted time vector

$$
\Omega^{i_{1} i_{2} \ldots i_{k}}=W^{i_{1} i_{2} \ldots i_{k}} \cdot T^{i_{1} i_{2} \ldots i_{k}} .
$$

The $i$ th component of the vector $\Omega^{i_{1} i_{2} \ldots i_{k}}$ gives a contribution to waiting time equal to $i$ coming from a path $i_{1}, i_{2}, \ldots, i_{k}$. Summing up those vectors for all possible paths we end with a distribution of waiting times. One can obtain also a distribution related to avalanches of chosen size. For example, if such sum is made for paths related to avalanches of size $2,3,4$, and 5 only, a distribution of waiting times related to avalanches of size bigger than 1 is obtained.

Rebound parameters presented in Table 2 were chosen in order to obtain noncumulative distribution of avalanches in the form consistent with Gutenberg-Richter law. The exact value of power (here 2.1) does not affect results of the construction substantially.

The system has average density $\rho=0.273885$, average avalanche size $\langle w\rangle=1.52458$ and average time between avalanches $T_{a v}=21.2027$. The parameter $P_{r}=0.880932$ shows that most of incoming balls are rebounded. Expected times of staying in all states are presented in Table 3. The great majority of avalanches leads to an empty state $\left(S_{1}=0.755449\right)$, roughly every fifth avalanche leads to state $2\left(S_{2}=0.205253\right)$, and roughly every twenty fifth to state $3\left(S_{3}=0.0392984\right)$.

Table 3

Approximate expected stay times in states for the size of the lattice $N=5$

\begin{tabular}{|l|c|c|c|c|c|c|c|c|}
\hline State & 1 & 2 & 3 & 4 & 5 & 6 & 7 & 8 \\
\hline$t_{a v}$ & 4.0 & 2.5 & 3.3 & 1.8 & 3.7 & 2.2 & 7.8 & 21.8 \\
\hline
\end{tabular}

Figure 2 presents obtained distributions of waiting times up to 300 time steps. The upper curve is for avalanches of all sizes, the next is for avalanches 


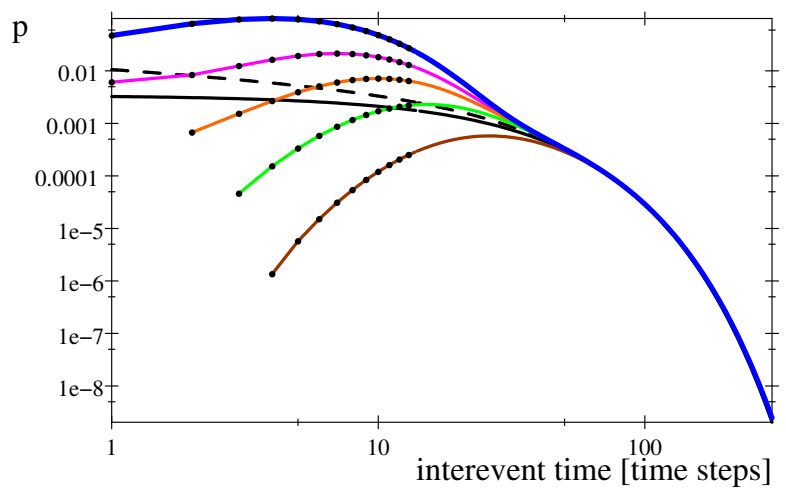

Fig. 2. A plot of distributions of interevent times for RDA with $N=5$. The upper line is for avalanches of all sizes, the curve below is for avalanches of size bigger than 1 , the next is for sizes bigger than 2 , and so on. The lowest curve counts only avalanches of size 5. The dashed line is a plot of fitted gamma distribution $a y^{(\gamma-1)} e^{-\frac{y}{b}}$; the solid line below is a plot of fitted exponential curve $a^{\prime} e^{-\frac{y}{b^{\prime}}}$. For large interevent times all these curves overlap.

of size bigger than 1 , and so on. The lowest curve counts avalanches of size 5 only.

The dashed line is a fitted gamma distribution $a y^{(\gamma-1)} e^{-\frac{y}{b}}$, where $\gamma=$ $0.67, b=22.4$, and $c=0.011$. This fit is done for points with time coordinate from $60\left(\chi^{2}=5.5346 \cdot 10^{-11}\right)$. Values of the parameters $b$ and $c$ can be rescaled, depending on their relation to physical quantities (time, number of earthquakes). The parameter $\gamma$ is a fixed parameter, with exactly the same value which characterizes Earth's seismicity. The solid line below is a plot of fitted exponential curve $a^{\prime} e^{-\frac{y}{b^{\prime}}}$.

Thus, the exponential part of the universal curve comes from distributions of biggest avalanches. In the presented example the biggest $t_{a v}$ is for the state 8 containing single cluster of size 5 (see Table 3 ). Thus its contribution to the overall waiting time distribution dominates for bigger times (compare formulas 6 and 7). Also state 7 containing single cluster of size 4 contributes, but it is decaying more rapidly.

The other part of the universal curve, comes from contributions of avalanches of smaller sizes. Its shape is a result of composition of many possible paths of the evolution, as depicted in Fig. 1. For bigger sizes $N$ there are much more possible paths (i.e., 1554 for $N=7$ ) through states containing many clusters with comparable times $t_{a v}$. Their composition flatten the curve. Moreover, calculation shows that this effect produces a surplus (comparing to the gamma fit) for small waiting times, which is evident in real earthquakes data 
(Corral 2004, Marekova 2012). The size of the surplus can be reduced by omitting of a contribution of smallest avalanches (also not recorded in real data).

Note that due to the incompleteness of the seismic catalogs in the short-time scale, real data are usually not displayed on plots for very short time intervals. Thus, the obtained theoretical curve, shown in Fig. 1, may be similarly cut for small times. If it is done for time, say, smaller then 10 , it reflects the shape of real data.

\section{CONCLUSIONS}

Thus, the presented model suggests that the origin of a universal curve is of combinatorial nature of accumulation and abrupt release of energy according to the rules depending on some parameters defining probabilities dependent on size of energy portions, as described above.

We would like to underline some limitations of the presented model resulting from its simplicity. Saichev and Sornette (2006) pointed out the importance of the mechanism of triggering of earthquakes by other earthquakes in understanding of distributions of inter-event times. Such mechanism is absent in the presented model, and we intend to introduce it. Another important issue is the comparison features of waiting time distribution in case of rescaled and not rescaled, and the appropriate scaling is another challenge for the model. Moreover, in the recent study Matcharashvili et al. (2015) investigated the relative ratio of correlated and uncorrelated waiting times, and they found that the ratio is similar for different catalogues. We send interested reader to this paper for a detailed discussion of properties of distributions of waiting times.

All this strongly suggests the need of extensions of the presented results in order to explain mechanistically the universal distribution of inter-event time for earthquakes.

Acknowledgment. This work was partially supported by National Science Centre, project 2012/05/B/ST10/00598. This work was partially supported within statutory activities No. 3841/E-41/S/2015 of the Ministry of Science and Higher Education of Poland.

\section{References}

Bak, P., K. Christensen, L. Danon, and T. Scanlon (2002), Unified scaling law for earthquakes, Phys. Rev. Lett. 88, 17, 178501, DOI: 10.1103/PhysRevLett.88. 178501.

Białecki, M. (2012), Motzkin numbers out of Random Domino Automaton, Phys. Lett. A 376, 45, 3098-3100, DOI: 10.1016/j.physleta.2012.09.022. 
Białecki, M. (2013), From statistics of avalanches to microscopic dynamics parameters in a toy model of earthquakes, Acta Geophys., 61, 6, 1677-1689, DOI: 10.2478/s11600-013-0111-7.

Białecki, M. (2015), Properties of a finite stochastic cellular automaton toy model of earthquakes, Acta Geophys. 63, 4, 923-956, DOI: 10.1515/acgeo-2015-0030.

Białecki, M., and Z. Czechowski (2010), On a simple stochastic cellular automaton with avalanches: simulation and analytical results. In: V. De Rubeis, Z. Czechowski, and R. Teisseyre (eds.), Synchronization and Triggering: From Fracture to Earthquake Processes, GeoPlanet - Earth and Planetary Sciences, Springer, Berlin Heidelberg, 63-75, DOI: 10.1007/978-3-642-12300-9_5.

Białecki, M., and Z. Czechowski (2013), On one-to-one dependence of rebound parameters on statistics of clusters: exponential and inverse-power distributions out of Random Domino Automaton, J. Phys. Soc. Jpn. 82, 1, 014003, DOI: 10.7566/JPSJ.82.014003.

Białecki, M., and Z. Czechowski (2014), Random Domino Automaton: Modeling macroscopic properties by means of microscopic rules. In: R. Bialik, M. Majdański, and M. Moskalik (eds.), Achievements, History and Challenges in Geophysics, GeoPlanet: Earth and Planetary Sciences, Springer Intern. Publ., Cham, 223-241, DOI: 10.1007/ 978-3-319-07599-0_13.

Corral, A. (2004), Long-term clustering, scaling, and universality in the temporal occurrence of earthquakes, Phys. Rev. Lett. 92, 10, 108501, DOI: 10.1103/PhysRevLett.92.108501.

Corral, A. (2007), Statistical features of earthquake temporal occurrence. In: P. Bhattacharyya and B.K. Chkrabarti (eds.), Modelling Critical and Catastrophic Phenomena in Geoscience, Lecture Notes in Physics, Vol. 705, Springer, Berlin Heidelberg, 191-221, DOI: 10.1007/3-540-35375-5_8.

Czechowski, Z., and M. Białecki (2012a), Three-level description of the domino cellular automaton, J. Phys. A: Math. Theor. 45, 15, 155101, DOI: 10.1088/1751$8113 / 45 / 15 / 155101$.

Czechowski, Z., and M. Białecki (2012b), Ito equations out of domino cellular automaton with efficiency parameters, Acta Geophys. 60, 3, 846-857, DOI: 10.2478/s11600-012-0021-0.

Drossel, B., and F. Schwabl (1992), Self-organized critical forest-fire model, Phys. Rev. Lett. 69, 11, 1629-1632, DOI: 10.1103/PhysRevLett.69.1629.

Marekova, E. (2012), Testing a scaling law for the earthquake recurrence time distributions, Acta Geophys. 60, 3, 858-873, DOI: 10.2478/s11600-012-0007-y.

Matcharashvili, T., T. Chelidze, and N. Zhukova (2015), Assessment of the relative ratio of correlated and uncorrelated waiting times in the Soutern California earthquakes catalogue, Physica A 433, 291-303, DOI: 10.1016/j.physa.2015.03. 060 . 
Saichev, A., and D. Sornette (2006), "Universal” distribution of inter-earthquake times explained, Phys. Rev. Lett. 97, 7, 078501, DOI: 10.1103/PhysRevLett.97. 078501.

Saichev, A., and D. Sornette (2013), Fertility heterogeneity as a mechanism for power law distributions of recurrence times, Phys. Rev. E 87, 2, 022815, DOI: 10.1103 /PhysRevE.87.022815.

Tejedor, A., J.B. Gomez, and A.F. Pacheco (2009), Earthquake size-frequency statistics in a forest-fire model of individual faults, Phys. Rev. E 79, 4, 046102, DOI: 10.1103/PhysRevE.79.046102.

Tejedor, A., J.B. Gomez, and A.F. Pacheco (2010), Hierarchical model for disturbed seismicity, Phys. Rev. E 82, 1, 016118, DOI: 10.1103/PhysRevE.82.016118.

Weatherley, D. (2006), Recurrence interval statistics of cellular automaton seismicity models, Pure Appl. Geophys. 163, 9, 1933-1947, DOI: 10.1007/s00024-0060105-3.

Received 20 March 2015

Received in revised form 13 July 2015

Accepted 11 August 2015 